\title{
Conocimiento social: Génesis y naturaleza de la representación de las figuras de apego
}

\author{
M. a José ORTIZ
}

Universidad del Pais Vasco

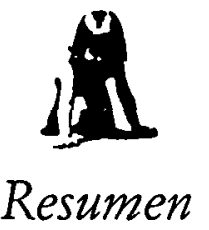

En este artículo se estudia la especificidad de los procesos de construcción de la representación de la figura de apego en el bebé y la naturaleza y función de este modelo interno en la adaptación al universo físico y social. Para ello, en primer lugar se analizan las teorias tradicionales, fundamentadas en la propuesta piagetiana sobre el desarrollo de la permanencia y la representación. A continuación se abordan los componentes sensomotores y emocionales de las unidades de interacción a partir de las cuales se construye el modelo interno de la figura de apego. La evolución de este proceso de construcción, partiendo del mero reconocimiento de recurrencia a la evocación de la representación de la figura materna, constituyente el objeto del tercer apartado. Por último, se presenta la noción de umodelos internos activos" propuesta por Bowlby, para conceptualizar este tipo especial de representación socioafectiva y su papel en la relación del niño con el entorno bumano y físico.

Palabras clave: Desarrollo cognitivo; Scripts; Apego; Primera infancia.

\section{Social knowledge: Genesis and nature of representation of attachment figures}

\section{Abstract}

This paper studies the specificity of the construction processes in the infant's representation of bis/ber attachment figure, and the nature and function of this internal model in the infant's adaptation to the physical and social universe. For this purpose, development of permanency and representation are analysed. The study then examines the sensory-motor and emotional elements present in episodes of interaction which the baby uses to build an internal model of the attachment figure. Third, the article analyses the development of this construction process; starting from the mere recognition of a recurrence in evoking the representation of the mother's figure. Finally, Bowlby's proposal of "Active Internal Models" is put forth in order to conceptualize this particular type of socio-afective representation together with its role in infants' relation with bis/ber buman and physical environment.

Keywords: Cognitive development; Scripts; Attachment; Infancy.

Correspondencia con autor-es: Universidad del País Vasco. Facultad de Psicología. Dpto. Procesos Psicológicos Básicos. Apartado 1249. Alto de Zorroaga s/n. 20080 San Sebastián.

Original recibido: Noviembre 1990. Revisión recibida: Febrero 1992. Aceptado: Mayo 1992. 
Es aceptada en la actualidad la estrecha relación entre el desarrollo cognitivo y el desarrollo socio-emocional; sin embargo, todavía los textos sobre psicología evolutiva presentan un abordaje unilateral de ambos procesos. Por otra parte, incluso en los numerosos esfuerzos de integración se observa un conflicto en el peso otorgado a los procesos individuales y sociales en la explicación del cambio evolutivo.

En el tema que nos ocupa, la construcción del conocimiento sobre la figura de apego, la necesidad de una perspectiva integradora se hace aún más necesaria. En este campo los esfuerzos por analizar la estrecha conexión entre ambas áreas han partido de los investigadores del desarrollo socio-afectivo. Son ya clásicos los primeros trabajos sobre los mecanismos cognitivos que subyacen en la ontogénesis de las emociones (Gouin Décane, 1962; Sroufe, 1979), en la evolución y en la organización del apego (Bell, 1970; Schaffer, 1971, 1974; Sroufe y Waters, 1977), las relaciones entre calidad del apego y niveles cognitivos (Bell, 1970; Bretherton y otros, 1979), etc. Desde el área del desarrollo cognitivo, aunque subsiste una importante dicotomía entre el modelo social y el individual, destaca el esfuerzo de numerosos investigadores por avalar la construcción social del conocimiento social en particular y del conocimiento en general (Vigotsky, 1962; Doise, 1978, Doise y Mugny, 1979; Nielson y Dockrell, 1982; Glachan y Light, 1982). Sin embargo en lo que se refiere a la génesis y naturaleza de la representación de la figura de apego la perspectiva estructuralista de Piaget continúa caracterizando las propuestas evolutivas.

El propósito de este artículo es analizar, en primer lugar, la capacidad explicativa de la aproximación piagetiana, centrándose fundamentalmente en la noción de permanencia y representación y en el paralelismo entre el conocimiento del mundo físico y social. En segundo lugar, se pretende defender y caracterizar la base interactiva en la construcción del conocimiento de la figura de apego, para finalizar describiendo la evolución de un tipo de representación «el modelo interno activo» construida a partir de la generalización de unidades interactivas relevantes para el sistema de apego.

\section{CUESTIONES A LA PROPUESTA PIAGETIANA}

La propuesta piagetiana afirma el paralelismo entre la construcción del mundo físico y el conocimiento social. Para este autor, este desarrollo solidario no es específico de estos dos procesos. La teoría de Piaget es uno de los ejemplos más representativos de la concepción unitaria del desarrollo. Con ella, se afirma la evolución paralela e interdependiente de la adaptación intelectual, la construcción de la realidad física, la construcción de las relaciones sociales, el desarrollo afectivo, la representación, la simbolización, etc. En consecuencia, aunque exista un desfase entre el conocimiento de las personas y el de los objetos, ambos se sitúan en el mismo plano.

Las investigaciones sobre la permanencia de las personas que, desde una línea piagetiana, han utilizado adaptaciones de la tarea de búsqueda, dan cuenta de una discrepancia temporal positiva a favor de la permanencia de la figura materna respecto a la de los objetos (Saint Pierre, 1962; Gouin Décane, 1962; Bell, 1970; Brossard, 1974). En el estudio realizado por Bell (1970), el 84\% de los niños mostró los mayores logros de desarrollo de la permanencia de la figura materna a los 13 meses y medio. Corroborando estos resultados, Brossard 
(1974) halló que a la edad de 9 meses y medio dos sujetos fueron situados en el estadio sexto y la mayoría accedieron al mismo a finales del primer año.

Considerando los datos aportados por los estudios citados, podríamos afirmar que las competencias representativas que subyacen en la permanencia de las personas son patentes ya a finales del primer año. Sin embargo, otro tipo de evidencias permiten retrotraer esta capacidad aún más, a los comienzos del segundo semestre. El apego del niño a la figura materna se ha establecido en los comienzos del segundo semestre del primer año. Uno de los criterios, mayoritariamente aceptado, de la formación del lazo es la ansiedad de separación. La protesta, la alteración emocional y los persistentes intentos de recuperar a la persona específica, consecuentes a la separación, implican la representación, la permanencia de la misma.

Aunque no se afirma que estas habilidades cognitivas sean suficientes para que la interacción se transforme en apego, estamos de acuerdo con Maccoby y Masters (1970), Schaffer (1971) Sroufe y Waters.(1977) y Bowlby (1980), entre otros, en que constituyen un soporte cognitivo necesario.

La capacidad de evocar a la figura de apego en su ausencia, manifiesta por los niños en el segundo semestre del primer año, plantea un importante problema a los psicólogos evolutivos. ¿Cómo explicar esta gran diferencia cronológica desde la formulación piagetiana, que afirma procesos semejantes para la construcción de la realidad física y la realidad social y en la que se afirma una asociación necesaria entre representación y simbolización?. Este interrogante nos lleva a cuestionar: o el nexo entre representación y simbolización, o el paralelismo entre el conocimiento del mundo físico y el de las personas, o bien ambas propuestas.

Podemos cuestionar ambos postulados. En primer lugar, existe suficiente evidencia para afirmar competencias representativas previas a la capacidad simbólica. En segundo lugar, los conocimientos actuales sobre la representación de objetos y personas permiten sostener que la diferencia entre ambas no consiste únicamente en una discrepancia temporal de dos procesos paralelos, sino que la distinción se sitúa en su diferente naturaleza, desarrollo y función. Y, en todo caso, serán estas diferentes cualidades las que expliquen la precocidad del conocimiento de las personas frente al del universo físico.

\section{Representación y simbolización}

Según Piaget, hasta el sexto estadio (18-24 meses), no existe representación mental. Aunque, sobre la base de los esquemas de acción y la percepción, admite la capacidad de reconocimiento de personas y objetos en etapas previas, como formas más sutiles de permanencia, la representación implica la evocación, la capacidad de actualizar un objeto o situación ausente. Y es preciso señalar que esta concepción asume un nexo necesario entre la función representacional y la simbólica y un origen en la acción para ambas.

Las primeras formas de representación en la formulación piagetiana son imágenes mentales, que no provienen de la percepción sino de una interiorización de la imitación. No quiere esto decir que la imagen aparezca de forma súbita, es el resultado de la imitación externa, sensomotora, que en el sexto estadio se prolonga en imitación diferida y en imitación interiorizada. Ambas constituyen los primeros significantes que permiten la evocación de objetos o realidades ausentes a la percepción actual. En el caso de los símbolos - imágenes, imitaciones 
diferidas (en sus variadas formas, desde la imitación gestual a la imitación gráfica) y juego simbólico - existe semejanza entre significante y significado, por la preponderancia de los aspectos figurativos. La diferenciación será completa con el empleo de los signos lingüísticos, no figurativos, arbitrarios, sociales.

Desde esta formulación, hasta el sexto estadio (18-24 meses) la inteligencia es únicamente sensomotora. Según Piaget, la representación del mundo externo, conocimiento o información capaz de ser evocado, implica una función simbólica, de forma que, en ausencia de símbolos, el niño puede conocer y reconocer la realidad a través de la acción, pero es incapaz de recordarla.

Sin embargo, disponemos de evidencia que permite situar en el tercer cuatrimestre este importante progreso de la memoria (Millar y Schaffer, 1973; Kagan, 1974; Ashmead y Perlmutter, 1980; Diamond, 1985, Meltzoff, 1988). También Flavell (1977) apunta hacia la existencia del recuerdo en épocas anteriores a las propuestas por Piaget:

«Parece plausible que pueda existir algún tipo de imagen mental primitiva antes del año y medio o los dos años, una especie de protoforma de representación y recuerdo consciente» (Flavell, 1977; pág. 206, de la traducción castellana).

Ahora bien, como apunta Mandler (1983), si el recuerdo exige simbolización, cabe proponer dos alternativas:

«.. éstos (los símbolos) deben aparecer bastante más tempranamente : o de otra manera, quizá la capacidad de simbolizar debe ser diferenciada de la habilidad para acceder a las representaciones de los objetos en su ausencia». (Mandler, 1983; pág. 474).

En torno a este tema es preciso citar un clarificador análisis realizado por Rivière (1984). El autor señala el problema de la circularidad entre representación y permanencia que genera la propuesta piagetiana:

«.la noción de permanencia de los objetos es, al mismo tiempo premisa y resultado de la capacidad de evocación simbólica. Resultado, porque implica representación (que se identifica con simbolización); premisa, porque no sería posible la construcción de símbolos sin unos significados referidos a objetos con una entidad sustancial». (Rivière, 1984; pág. 157).

En su opinión, esta paradoja deriva de la indiferenciación entre representación como capacidad de evocación y mantenimiento en la conciencia de esquemas o modelos internos, y simbolización, entendida como la capacidad de convertir la información interna en formas observables, con una doble función representacional y comunicativa.

Rivière, tras revisar los principales datos que sugieren competencias representativas precoces (las manifestaciones tempranas de permanencia, el reconocimiento visual de objetos explorados previamente a través de otra modalidad sensorial, la capacidad de anticipar y planificar la acción, etc.), aunque no apoya la hipótesis de una capacidad de representación innata, considera que estos fenómenos demuestran un desarrollo temprano de la misma, que permite la construcción de lo real en ausencia de capacidad simbólica. La necesidad de diferenciar ambas competencias es también apoyada por los estudios que han analizado la relación entre diferentes competencias cognitivas. Rivière (1984) refiere el trabajo realizado por Bates y colaboradores (1979) en el que se hallaron elevadas correlaciones entre el desarrollo de la comunicación y los índices tempranos de imitación, juego y comportamiento instrumental. Por el contrario, los índices de desarrollo simbólico mostraban escasa relación con los de permanencia y comprensión espacial. 
Si aceptamos tal distinción, desde nuestro punto de vista, es posible considerar la existencia de competencias representativas en el tercer cuatrimestre del primer año, mientras se mantiene la emergencia de la función simbólica, representativa y comunicativa, en los últimos momentos del período sensoriomotor.

\section{El paralelismo entre la objetivación del mundo físico y social}

Existen suficientes razones para inferir procesos diferentes en la construcción de la representación de objetos y personas y sobre ello nos detendremos más adelante. Pero incluso prescindiendo de los actuales conocimientos sobre el tema, son evidentes las limitaciones derivadas de una estricta aplicación del proceso piagetiano de construcción del mundo físico al universo social.

Durante el período sensomotor el niño comprende los objetos, sus estados y transformaciones a partir de sus atributos físicos y, sobre todo, a partir de las características de su propia acción sobre ellos. Como señalan Gelman y Spelke (1981), aunque los seres humanos evidentemente comparten con las cosas propiedades físicas, actúan por sí mismos y reaccionan a la acción del niño; además, no lo hacen sólo según leyes físicas, sino sobre todo de acuerdo a procesos psicológicos internos y costumbres sociales. Por ello, es cuestionable que el sistema de conocimiento relativo al mundo social se rija por las mismas leyes de coordinación y regulación de la acción que permiten la construcción del mundo de los objetos.

En lo que concierne al desarrollo de la permanencia, Gelman y Spelke (1981) dan cuenta de algunas dificultades:

Según Piaget, el desarrollo del concepto de permanencia requiere que el niño aprenda que la existencia de los objetos es independiente de su actividad motriz o perceptiva y su construcción se evidencia en los progresos en la conducta de búsqueda de objetos desaparecidos.

Aunque la acción sobre los objetos y sobre las personas fuera semejante, la reacción de estas últimas es menos predecible, no siempre está en función de cómo se actúe sobre ellas. Mientras en los desplazamientos de los objetos sólo cabe predecir una causa externa, los seres humanos son autónomos: el objeto permanecerá en el lugar donde se ocultó, pero es mucho más probable que la madre que desaparece tras la puerta no mantenga esta posición. Por ello, la atención a los desplazamientos o transformaciones provocados por acciones externas tendrá menor capacidad predictora en la localización de las personas que el conocimiento de sus hábitos y rutinas.

Por otra parte, la recuperación del objeto escondido supone realizar la transformación inversa a la que ha producido su ocultación. Una coordinación de medios y fines permite al niño eliminar el obstáculo. Pero en la desaparición de las personas la transformación es provocada por ellas mismas, y la recuperación no es siempre una acción directa del niño. De hecho, la actividad del niño consiste habitualmente en promover a través de sus señales comunicativas que la misma figura de apego invierta la transformación.

Con estas consideraciones no se pretende negar cierta precocidad de la representación de las figuras de apego sobre la de los objetos inanimados, sino destacar la dificultad de extrapolar el proceso de construcción de la realidad, tal como lo formula Piaget, al desarrollo del conocimiento de las personas.

Una vez realizadas las puntualizaciones referentes al nexo representación simbolización y a las limitaciones que derivan del pretendido paralelismo entre 
el conocimiento de los objetos y el de las personas, resta abordar el punto fundamental de esta exposición: la construcción por parte del lactante de la representación de las figuras de apego. Para ello, presentaremos primeramente los aspectos que aportan la especificidad a la relación con las personas significativas y que nos permiten afirmar que los elementos a partir de los cuales se constituye este mundo representacional no son esquemas de acción sino experiencias interactivas. A continuación, intentaremos abordar la construcción del modelo interno de la figura de apego a partir de las unidades básicas de interacción. Finalmente, describiremos la específica naturaleza y función de la representación de la experiencia interactiva con esta figura privilegiada.

\section{UNIDADES INTERACTIVAS BASICAS}

En las páginas que siguen se presenta la especial experiencia del niño con las personas que le cuidan. Este intercambio es especial, fundamentalmente porque, mientras en la relación con los objetos inanimados podemos distinguir la experiencia sensorial del objeto y la experiencia motora de la acción realizada por el niño, en la interacción con las figuras de apego interviene fuertemente un tercer elemento: la experiencia emocional del bebé. Pero además, dadas las características y la función del comportamiento interactivo, también las experiencias sensoriales y motoras relacionadas con las personas que cuidan al niño difieren significativamente de las correspondientes a los objetos.

Los conocimientos sobre las competencias del bebé, aportados por la investigación sobre el procesamiento de la información y por la extensa serie de trabajos sobre el apego, permiten afirmar que el niño viene al mundo dotado, por una parte, de una sensibilidad preferencial por los estímulos de origen social, hacia los que se orienta activamente, y, por otra, de un un sistema de señales (sonrisa, llanto, expresiones emocionales) y conductas (prensión, aferramiento, succión no nutritiva, etc.) cuya meta es la proximidad, el contacto y la interacción con las personas que le cuidan. Estas, a su vez, también le ofrecen señales y conductas privilegiadas. Las figuras de apego manifiestan una generalizada tendencia a proporcionar las entradas sensoriales óptimas, a conferir significado a las señales y conductas del niño y a responder adecuada y contingentemente. Es unánimemente aceptado que estas disposiciones, seleccionadas a lo largo de la evolución de la especie, promueven el establecimiento del vínculo afectivo, garantizándose la protección y la supervivencia del bebé.

Asumir la función supervivencial del apego y afirmar su carácter primario haciendo referencia a disposiciones de carácter biológico no quiere decir que este lazo exista desde el nacimiento. Debemos aceptar que en el establecimiento, en la organización y en la evolución del apego también intervienen la experiencia y el desarrollo emocional y cognitivo. Logros evolutivos como la discriminación de familiares y extraños, la capacidad de evocación, la planificación del comportamiento, etc., son prerrequitos cognitivos para que, en los comienzos del segundo semestre de la vida, la interacción se transforme en relación y el sistema de apego se organice como jerarquía planificada y flexible de conducta dirigida a una figura específica.

Pero debemos adoptar también una perspectiva integradora cuando abordamos los procesos por los los cuales la experiencia interactiva pasa a formar parte del conocimiento. Las predisposiciones genéticas van a jugar un rol fundamen- 
tal en la génesis y desarrollo de la representación de la figura de apego. Por una parte, los episodios interactivos determinados por motivaciones fundamentales, como el apego, tienen un carácter repetitivo, lo que les confiere cierta prominencia sobre el resto. Por otra, como se expondrá próximamente, el sistema de apego no sólo orienta la percepción y controla la conducta, sino que también interviene, en su vertiente subjetiva, emocional, en la evaluación de la experiencia interactiva, en el significado que se le confiere a la misma. Este componente emocional determina también la pregnancia de ciertas unidades interactivas.

\section{Experiencia sensorial privilegiada con las personas}

Entre las razones que pueden explicar la prominencia de los compañeros humanos respecto a cualquier elemento inanimado del entorno es preciso destacar, en primer lugar, la preferencia perceptiva por los estímulos de origen social, manifiesta en los niños desde los primeros momentos de la vida. La investigación sobre las capacidades perceptivas y el procesamiento de la información en la infancia permite considerar al niño como un ser activo, con capacidad de regular la información entrante a través de una atención selectiva, desde los momentos iniciales de su existencia.

Los trabajos iniciales realizados por Fantz $(1961,1966)$, utilizando la técnica de la preferencia visual, y posteriores investigaciones sobre la atención visual selectiva (Spears, 1964; Brennan, Ames y Moore, 1966; Fantz, Fagan y Miranda, 1975; Gouin Décarie y Ricard, 1983) han posibilitado descubrir las propiedades estimulares más eficaces para provocar una conducta visual diferencial. Entre ellas destacan las siguientes: contorno redondeado, movimiento, brillo, tridimensionalidad, complejidad, contraste, estructuración. Y es precisamente el rostro humano la configuración estimular que posee tales características distintivas. Lo cual sugiere que los lactantes están biológicamente preparados para orientarse hacia los miembros de su especie.

Respecto a la sensibilidad auditiva, también parece encontrarse una preadaptación semejante. Las características del lenguaje humano generan una clara preferencia por el mismo desde los primeros momentos de la vida. Lactantes de 3 y 8 días manifestaron registros eléctricos musculares más intensos ante la voz humana o sonidos semejantes a ésta en estructuración y frecuencia (Hutt, Hutt, Lenard, Bernuth, y Muntjewerff, 1968). La preferencia por la voz humana es también constatada por Eisenberg (1976), Mehler y otros (1978) y Lecanuet y otros (1987). Los estudios de De Casper (1980) utilizando el método de la succión no nutritiva, van todavía más lejos al observar que, ya en el tercer día de la vida, los niños son capaces de discriminar la voz de su madre de la de otra mujer desconocida.

Por último, hay que mencionar los trabajos sobre la precocidad de la discriminación olfativa. A la edad de 10 días, 25 de los 32 niños observados por MacFarlane (1975) mantenían durante más tiempo su cabeza vuelta hacia un algodón que había estado en contacto con el pecho de su madre. Los datos aportados por Russell (1976) y Montagner (1988), entre otros, corroboran la discriminación significativa del olor corporal materno frente a otros estímulos olfativos a los pocos días de nacer.

La selección perceptiva hacia tales atributos relevantes característicos de las personas, permite otorgar a éstas un rol especial como configuración estimular. Esta preferencia sensorial por los seres humanos sugiere una motivación endó- 
gena para el aprendizaje perceptivo de los rasgos distintivos de las figuras de apego. Nos aproxima a la consideración de un troquelado concebido como desarrollo de la familiarización, tal como lo formula Hinde (1966), que permite la distinción, biológicamente ventajosa, entre objetos y personas, gracias a los modelos centrales construidos en este precoz y programado encuentro perceptivo.

Pero además, es preciso destacar el rol especial de las figuras parentales al desarrollar un tipo muy particular de interacción con los niños: íntima, desformalizada, asimétrica, repetitiva, sincronizada, contingente, etc. Las expresiones maternas en la interacción con el niño llaman la atención, en primer lugar, por la repetición y exageración espacial de aquellos rasgos o elementos propios de cada una de ellas, lindando muchas veces con la caricatura, lo cual facilitará el reconocimiento. También es característica una exageración temporal, de acuerdo a la capacidad de procesamiento de la información del lactante, y cabe suponer que este enlentecimiento de las variadas transformaciones faciales maternas posibilitará al niño captar la constancia. Por otra parte, un elemento importante en la experiencia sensorial con la madre es la transformación continua: madre sonriente, seria, con gafas, sin ellas, con diferentes peinados, etc. Pero, dado que la discrepancia es moderada, le proporciona también información de una constante. El niño tiene posibilidades de detectar los elementos invariables que pertenecen a la figura de apego, a pesar de las modificaciones de su apariencia. Por último, la figura de apego no sólo se adapta a las capacidades de procesamiento de la información del niño, sino que regula el estado emocional de éste, para permitir y favorecer la entrada y el procesamiento de la información.

\section{Experiencia motora versus comunicativa.}

El elemento diferencial por excelencia de la experiencia motora infantil con las personas, frente al mundo de los objetos, es el de provocar respuestas sociales. Aunque durante los primeros meses la actividad infantil no es dirigida ni intencional, los adultos le confieren significado e intención. Así, si el feedback de la acción sobre los objetos es un resultado interesante, de las personas recibe una respuesta contingente, lo cual le permite comprender que ciertas actividades tienen valor de señal y de establecer expectativas sobre el comportamiento de las personas con las que interactúa.

Retomando el tema de la precocidad de las competencias cognitivas referidas a las figuras de apego, debemos considerar, además, que si en relación con el mundo físico, durante los primeros meses la acción consiste en movimientos centrados en sí mismo, las personas que cuidan al niño consideran como gestoß significativos acciones que de otra manera serían autísticas. Además, como se señaló anteriormente, el niño viene equipado con un repertorio de disposiciones (sonrisa, llanto, vocalización, expresiones emocionales) orientado a inducir y regular la interacción. Estas reacciones, pertenecientes al sistema de señales, altamente organizado desde épocas tempranas de la vida, preceden al desarrollo de otro tipo de habilidades motrices y no se dirigen a la asimilación o incorporación del objeto. Su función primordial es organizar el comportamiento materno, en virtud de su capacidad comunicativa. Tales premisas sugieren que la orientación hacia el entorno humano precede al interés por los objetos y que el significado de las personas no está tan relacionado con la asimilación a esquemas de acción, sino con las consecuencias de la misma. Esto es, con la adecua- 
ción de la respuesta de los compañeros humanos a la actividad infantil dirigida a la búsqueda de proximidad e interacción.

Otra importante base para acreditar el carácter comunicativo de la actividad del lactante proviene de los estudios sobre la conducta diferencial ante objetos y seres humanos.

Brazelton y otros (1974) analizaron comparativamente el comportamiento del bebé durante las tres primeras semanas de la vida ante un objeto físico (un peluche) y la interacción con la madre, observando una clara diferenciación en la respuesta. Ante el objeto los niños reaccionaron con una atención prolongada, movimientos irregulares y finalización brusca, mientras que hacia la madre dirigieron la atención en fases más cortas, cambiando suavemente de la atención a la desatención y mostraron un mayor control de la actividad. Trevarten (1977, 1979), a partir de las diferencias observadas en la expresión facial, movimiento de las manos y vocalización ante la madre y los objetos en el comienzo del tercer mes, sugiere dos tipos de comportamiento: acción (con los objetos) y comunicación (con las personas).

Si la orientación y la conducta infantil hacia los seres humanos y la respuesta de éstos evidencian la comunicación desde épocas tempranas ésta será, además, facilitada por otra importante disposición infantil. Nos referimos a la organización temporal de ciertos patrones de comportamiento. El ritmo estable facilita a la figura materna anticipar o prever la conducta del niño y las pausas le permiten ejercer un rol activo, estableciéndose una sincronía interpersonal. En relación con esta precoz sincronía en la interacción, es preciso señalar los estudios realizados por Kaye $(1972,1977)$, Kaye y Wells (1980) sobre la adaptación mutua en el amamantamiento. En esta situación se observó una especial alternancia de roles, expresada en la actividad manipulativa de la madre durante las pausas y la reactivación de la succión por parte del niño cuando cesaba la actividad materna. Esta organización temporal succión-pausa, específicamente humana, innecesaria para la alimentación, es interpretada por los autores como una preadaptación infantil que permite el desarrollo de un intercambio de roles (turn-taking), fundamental en una especie como la nuestra, que puede y precisa comunicarse.

Respecto a la regulación del intercambio vocal, Bateson $(1975,1979)$ dio cuenta también de una estructura de alternancia, al observar la interacción madrehijo en el segundo y tercer mes, definiéndola como «proto-conversación».

\section{Experiencia emocional}

A lo largo de su exposición sobre el desarrollo de la inteligencia y la construcción de lo real Piaget omite la determinación social-afectiva del objeto de conocimiento. Mientras en las observaciones particulares los objetos son concretos y con significado cultural, social y afectivo, a nivel teórico y conceptual parece referirse a objetos formales.

El significado del objeto lo aporta la asimilación al esquema, interno o externo. Ahora bien, aunque para este autor, afectividad y cognición son dos aspectos inseparables de la acción real o posible, el papel otorgado al componente afectivo es de carácter energético, y es la inteligencia la que proporciona la estructura. Asumir que la organización mediadora subyacente a cualquier comportamiento social, intelectual y afectivo es siempre una estructura de conocimiento, supone que, tanto el significado de los objetos, como el de las 
personas es de carácter cognitivo, ya que, para Piaget, la asimilación social no difiere de la asimilación del objeto físico.

No obstante, la experiencia motora y la experiencia sensorial en la interacción con las figuras de apego están a su vez inevitablemente ligadas a modificaciones del nivel de excitación, a variadas sensaciones internas y emociones. Los diferentes estados de excitación placentera o desagradable son tanto producto, como causa inmediata de la interacción. Stern justifica el interés en los cambios emocionales:

«...en primer lugar, estos momentos son los que poseen más probablemente un alto valor como estímulos (en virtud del contraste) y, en segundo lugar, la duración temporal y la naturaleza de tales momentos nodales es más probable que corresponda a aquello que he designado como unidades procesuales interpersonales.»

(Stern, 1977, pág. 166 de la traducción castellana).

Sólo disponemos de pruebas indirectas que demuestren que el investimento emocional favorezca la construcción de modelos internos. No obstante, se puede inferir que el componente afectivo determinará la prominencia de ciertas unidades de interacción sobre otras a la hora de ser almacenadas en la memoria. Con esta selección, mientras unos episodios, son relegados, aquellos relevantes desde el punto de vista afectivo constituirán las unidades básicas de la construcción de un prototipo de experiencia interactiva. Por otra parte, sí puede aceptarse la implicación del afecto positivo en la memoria (Isen, Shalker, Clark y Karp, 1978). Es posible que la precocidad referida a la permanencia de las figuras de apego se relacione también con la mayor facilidad para evocar una representación investida emocionalmente.

Como resumen de lo que acabamos de exponer acerca de las unidades interactivas, podemos decir, siguiendo a Olson (1981), que las personas como fuentes de conocimiento proporcionan dos tipos de información: perceptiva y disposicional.

- Los seres humanos, como elementos de percepción, poseen una serie de características estimulares especialmente relevantes para la orientación perceptiva infantil que les otorga una clara prominencia sobre los objetos.

-En segundo lugar, a diferencia de los objetos, ofrecen una importante información disposicional. El carácter frecuente, variado y multisensorial de la estimulación, su adaptación a las capacidades de procesamiento de la información infantil, la sincronización con los ritmos temporales del niño, la respuesta a sus señales y acciones, etc, son propiedades que ejercen un significativo papel en la cognición social. Y, fundamentalmente, a partir de las reacciones de las figuras de apego a lo largo de esta intensa y rica experiencia interactiva se generan expectativas. La confirmación o falta de confirmación de estas expectativas, según Olson, proporciona también una importante base del reconocimiento:

«..aunque las características perceptivas de las personas generalmente definen las condiciones que inician el reconocimiento, la confirmación o falta de confirmación de expectativas puede proporcionar una posterior base para el reconocimiento que podría determinar significativamente la respuesta final del niño»

(Olson, 1981. pág.41). 


\section{LA REPRESENTACION DE LA FIGURA DE APEGO. EVOLUCION.}

Es muy probable que las unidades interactivas (sensomotoras-emocionales) inicialmente se interioricen y almacenen en la memoria de manera aislada y que progresivamente se organicen e integren hasta lograr una comprensión o modelo unificado de la figura materna en el que confluyen los elementos perceptivos, por una parte, y la generalización de experiencias asociadas o propiedades disposicionales, por otra.

Es acertada la progresión que presenta Olson (1981) durante el primer año de la vida para el desarrollo del concepto de persona, partiendo del reconocimiento perceptivo de la mera recurrencia.

Reconocimiento. Para este autor, el proceso básico del reconocimiento es la conexión entre un estímulo perceptivo y un rasgo almacenado en la memoria y fruto de esta comparación se produce la activación de otros rasgos asociados. Pero es preciso diferenciar dos tipos de reconocimiento, que caracterizan diferentes momentos evolutivos: reconocimiento de la recurrencia y reconocimiento de la identidad categorial y disposicional.

1) Según Olson, durante los tres primeros meses de la vida lo que el niño reconoce es la mera recurrencia de ciertos aspectos perceptivos de la madre (reconocimiento de que algo se ha experimentado anteriormente) y, por ello, otras personas son erróneamente reconocidas como la madre, ya que no existe aún un esquema de la misma.

2) Un proceso más complejo es reconocer que determinada experiencia pertenece o es un ejemplo de una categoría.

Como afirma Palacios (1984), tras revisar la investigación sobre procesamiento de la información en la infancia, existen pruebas suficientes para afirmar que durante el segundo trimestre de la vida los niños son capaces de formar categorías o prototipos de la experiencia.

Considerando las especiales cualidades de la interacción con la figura materna es aceptable que el establecimiento de un esquema prototipo de la madre preceda a otro tipo de categorías. Los estudios sobre discriminación visual entre familiares y extraños (Ambrose, 1961; Yarrow, 1967; Cornell, 1974; Olson, 1981) permiten situar alrededor del cuarto mes la formación de un modelo central de la figura de apego. Incluso, según los resultados presentados por Barrera y Maurer (1981), este esquema prototipo que permite detectar las diferencias de los rasgos de otras personas, se puede retrotraer al tercer mes.

Pero además, de acuerdo con Olson (1981), aunque son los atributos perceptivos los que permiten el reconocimiento, una vez que la madre es individualizada y reconocida como miembro de una categoría es predecible la consecuente activación de un rico conocimiento disposicional. El reconocimiento perceptivo generará expectativas respecto a la inmediata experiencia o posterior reacción de la figura familiar. En otras palabras, estamos hablando de un rasgo de memoria prototipo de la experiencia de interacción, una categoría elaborada a partir de los episodios, de las secuencias interactivas realmente experimentadas.

En cuanto al desarrollo del conocimiento disposicional en los lactantes, la evidencia empírica (Whaler, 1967; Carpenter, 1975; Cohn y Tronick, 1983; Field, 1984) confirma la hipótesis de que la formación del prototipo perceptivo de la figura de apego va ligada a un tipo de experiencias o consecuencias. Carpenter (1975) observó reacciones de desasosiego ante el comportamiento inusual de la 
figura materna. Cuando ésta se limitaba a mirar al niño, sin sonreír o moverse, la inquietud infantil era evidente. Estudios más recientes (Cohn y Tronick, 1983; Field, 1984) han constatado también una importante modificación del comportamiento infantil (evitación de la mirada, llanto, manifestaciones de protesta, desorganización conductual, etc.) en los primeros meses de la vida, cuando, a instancias del investigador, la madre interrumpe sus sonrisas, vocalizaciones y movimientos faciales, para adoptar una expresión rígida o mostrarse deprimida.

Field (1984) se propuso analizar, además, si a la edad de tres meses los niños cuyas madres padecían una depresión post-parto reaccionaban de la misma manera que los demás en una situación experimental en la que se invitaba a la madre a «parecer deprimida» (inexpresiva, quieta, utilizando un tono monótono en el lenguaje). Sus observaciones apoyan la idea de que ya a esta edad los bebés disponen de expectativas respecto al comportamiento materno. Los niños del grupo control, comparados con los hijos de madres deprimidas, mostraron más expresiones faciales negativas, más miradas recelosas, mayor protesta y menor incidencia de expresiones faciales y vocales positivas durante la situación «deprimida» versus «interacción espontánea».

Ahora bien, la existencia de este modelo (perceptivo y disposicional) permite el reconocimiento, pero no supone una concepción de la madre como permanente. En la primera mitad del $10^{\text {er }}$ año, el niño no parece tener conciencia de la existencia independiente de la figura materna, ni echarla de menos en su ausencia. Es como si este modelo sólo fuera activo o presente en conexión con la estimulación correspondiente.

Evocación. La hipótesis de que el paso del reconocimiento al recuerdo de las figuras de apego se produce en los comienzos del segundo semestre, ya propuesta por la teoría psicoanalítica, muestra una importante aceptación en la actualidad (Bowlby, 1969; 1980; Schaffer, 1966; 1971; 1974 ; Kagan, 1974; 1984.)

Los estudios sobre el desarrollo de la utilización del recuerdo en la búsqueda del objeto en la tarea $\mathrm{AB}$, han demostrado que alrededor del $8 .^{\circ}$ mes los niños son capaces de evocar la experiencia previa, cuando el lapso de tiempo transcurrido entre la ocultación y recuperación se limita a escasos segundos. En los últimos meses del primer año la capacidad de relacionar la experiencia pasada con la presente abarca progresivamente períodos más extensos (Kagan, 1974; Kagan, Kearsley y Zelazo, 1978; Diamond,1983; 1985). Si esto es cierto para el mundo físico, en lo que se refiere a la realidad social, no sólo se puede predecir que en esta época el niño dispone de una representación de la figura de apego, sino que además ésta será más activa y precoz, dado que los elementos investidos emocionalmente son evocados con mayor facilidad (Isen, Shalker, Clark y Karp, 1978).

Resta ahora intentar caracterizar este tipo de representación de la figura de apego, su naturaleza y función en los últimos meses del primer año y comienzos del segundo.

\section{«INTERNAL WORKING MODELS»}

Considerando el proceso de desarrollo de la representación de la figura de apego en un marco interactivo, que responde en buena parte a repertorios de conducta ambientalmente estables y que genera un conocimiento tanto perceptivo como disposicional, cabe predecir que el modelo interno presentará unas 
características especiales y funciones claramente distintas a las que definen la representación del mundo físico.

Es muy acertada la noción de «internal working model», formulada por Bowlby (1969) y desarrollada más recientemente por Bretherton y Waters (1985) y Main, Kaplan y Cassidy (1985). En líneas generales, este concepto hace referencia a una representación, que incluye componentes cognitivos y afectivos, y que se construye a lo largo del desarrollo a partir de la generalización de acontecimientos relevantes para el sistema de apego. Una vez formada, se integra a su vez en este sistema de conducta, ejerciendo un papel activo en la evaluación de la experiencia y en la orientación de la conducta.

Este tipo de representación social no es un concepto abstraído a partir de las características estáticas del entorno, o una categoría derivada de las similitudes y diferencias, sino que se relaciona más con las señales y conductas de apego y las consecuencias sociales de las mismas:

«El modelo interno activo de la relación con la figura de apego no reflejará una imagen objetiva de «la figura parental» sino la bistoria de las respuestas del cuidador a las acciones o tentativas de acción del niño con / hacia la figura de apego».

(Main, Kaplan y Cassidy, 1985, pág. 75).

Este modo de concebir la representación de la figura de apego tiene su origen en el modelo de «scripts» propuesto por Schank y Abelson en 1977. En dicha formulación, el «script», nivel básico de representación, se refería a una secuencia de interacción social típica, de acciones relacionadas temporal y causalmente. Según Bretherton (1985), el «internal working model» de la figura de apego es una generalización de las unidades interactivas, de acciones y consecuencias.

Su adecuación a la cognición social es evidente. El conocimiento sobre las personas y sus relaciones se adquiere a través de la participación en experiencias interactivas, manifiestas desde los primeros momentos de la vida. Entre las características de estas primarias unidades de interacción cabe señalar la ordenación temporal y la alternancia de roles. Además, como ya se expuso en el apartado anterior, la respuesta contingente de las figuras parentales a la actividad infantil da lugar al conocimiento disposicional, a la formación de expectativas. $Y$ finalmente, recordando la descripción de las unidades interactivas, podemos predecir que en la construcción del modelo interno de la figura de apego, participarán fundamentalmente aquellos episodios de interacción relevantes por su relación con el sistema de apego y destacados en virtud de su componente emocional. En este sentido, se propone una interdependencia entre el modelo activo de la figura de apego y el modelo de sí mismo, de tal forma que sería más adecuado hablar de un modelo de la relación.

Otro aspecto a destacar en este modo de concebir la representación de la figura de apego se refiere a su función. Un modelo interno como generalización de unidades interactivas relevantes con la figura de apego, ejercerá un rol determinante en la evaluación de las determinadas situaciones y, por tanto, en la orientación del comportamiento.

En este sentido hay que mencionar la iniciativa de Main, Kaplan y Cassidy (1985) de abordar las diferencias individuales en la seguridad del apego en términos de «modelos internos».

Seguridad del apego-Modelos internos. A partir del balance apego-exploración y de las reacciones ante una breve ausencia y posterior retorno de la figura ma- 
terna, evaluados en «la situación extraña», Ainsworth, Blehar, Waters y Wall (1978) diferenciaron tres patrones de apego en una dimensión seguridadinseguridad:

Apego seguro (B ). Los niños clasificados en este grupo (66\%), en presencia de la madre, orientaban su conducta hacia la exploración del entorno; la ausencia, provocaba un sensible decline de la actividad exploratoria y ansiedad, aunque esta última no era necesariamente intensa y la búsqueda de proximidad y contacto caracterizaba su respuesta en el reencuentro.

Apego inseguro-ambivalente $(C)$. Para los niños clasificados en este grupo (12\%) la figura materna no constituía una base de exploración; la inseguridad, ya manifiesta en presencia de la madre, era extremadamente intensa en los episodios de separación, y la ambivalencia ante su vuelta constituía la reacción más frecuente.

Apego inseguro-rechazante (A). Aproximadamente el 20\% de los niños fueron clasificados en este grupo. La falta de evidencia de inquietud en los episodios de separación y la tendencia a ignorar a la figura de apego y a evitar la proximidad y contacto con ella durante el reencuentro definían este patrón.

Ainsworth y otros (1978) analizaron la relación entre los diferentes patrones de apego y el tipo de interacción madre-hijo, observada en el hogar. La sensibilidad y responsividad a las señales infantiles y el tono positivo en la interacción fueron mayoritariamente manifiestas entre las madres del grupo B. Los patrones de apego inseguro se relacionaron con una falta de sensibilidad materna, que en el grupo A se combinaba, además, con rechazo y evitación.

$\mathrm{Si}$ asumimos que la seguridad del vínculo se relaciona con la experiencia de interacción previa y que el modelo interno se construye a partir de una generalización de unidades interactivas, podemos concebir, como proponen Main y otros (1985) y Bretherton (1985), las diferencias individuales en la organización del apego como diferencias en la representación del mismo.

Una historia de interacción caracterizada por la sensibilidad y la consistencia de la responsividad materna ante las señales y conductas infantiles generará una representación de la figura de apego protectora y un modelo de sí mismo como digno de amor. El niño puede anticipar la accesibilidad y ayuda de la madre y su propia competencia como promotor de la proximidad e interacción. Por el contrario, la anticipada ausencia de respuesta por parte de la figura de apego (modelo de figura de apego insensible o rechazante) confiere al niño la responsabilidad de la protección, desarrollándose un modelo de sí mismo como indigno o incapaz de promover cuidado, ayuda y amor. En ambos casos diferirá la orientación de la atención, la evaluación de las distintas situaciones y experiencias, los sentimientos y la conducta, tanto si es dirigida al mundo social, como al universo físico.

A modo de conclusión. La construcción de la representación de la figura de apego es uno de los procesos en que experiencia, cognición, afecto y disposiciones ambientalmente estables están más estrechamente relacionados. La base de esta construcción son las unidades de interacción, secuencias de acciones relacionadas temporal o casualmente, cuyo carácter constante y repetitivo permite el establecimiento de un modelo de la experiencia de intercambio con el que comparar la interacción actual. Posteriormente, con el desarrollo de la capacidad de recuerdo este modelo interno puede ser evocado en ausencia de la estimulación correspondiente. Ahora bien, es preciso destacar la intervención en este proce- 
so de las predisposiciones de carácter hereditario. Por una parte, estas tendencias orientan la percepción, facilitan el establecimiento de patrones temporales (acción-pausa) entre el niño y el cuidador, y regulan las acciones y reacciones de ambos. Pero además, cabe suponer que estas disposiciones ejercen también un papel relevante en la construcción del modelo interno. De entre la inmensa gama de unidades interactivas, aquellas que son reguladas por tendencias ambientalmente estables, como el apego, tienen un carácter repetitivo y muchas de ellas un tinte, un significado emocional. La repetición crea patrones de interacción, secuencias típicas, y el contraste emocional, los destaca sobre el resto.

En este sentido es coherente concebir el modelo interno de la figura de apego como una representación construida a partir de la generalización de acontecimientos relevantes para el sistema de apego. A su vez, este modelo interno de la relación, que incluye componentes cognitivos y afectivos, pasa a formar parte del sistema de apego, ejerciendo un rol determinante en la evaluación de la experiencia y en la orientación de la conducta.

\section{Referencias}

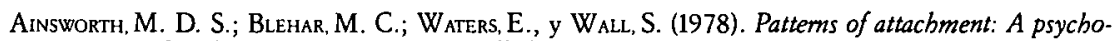
logical study of the strange situation. Hillsdalle, N.J.: L.Erlbaum.

Ambrose, J. A. (1961). The development of the smiling response in early infancy. En B.M. Foss (Ed.) Determinants of Infant Behaviour, Vol. 1. Londres: Methuen.

Ashmead, D. H., y Perlmutter, M. (1980). Infant memory in everyday life. En M. Perlmutter (Ed.) New Directions for Child Development: Children's memory. Vol. 10. Jossey - Bass: San Francisco.

Barrera, M. E., y Maurer, D. (1981). Discrimination of strangers by the three-month- old. Child Development, 52, 558-563.

Bates, E.; Benigni, L.; Bretherton, T.; Camaion, L., y Volterra, V. (1979). The emergence of symbols: Cognition and comunication in infancy. Nueva York : Academic Press.

Bateson, M. C. (1975). Mother-infant Exchanges: The Epigenesis of Conversational Interaction. En D. Aronson y R.W. Ruber (Eds.) Developmental Psycholinguistics and Communication Disorders. Nueva York: Academy Press.

Bateson, M.C. (1979). The Epigenesis of Conversational Interaction : A personal account of researh development. En M.Bullowa (Ed.) Before Speech: The beginning of Interpersonal Communication. Cambridge: University Press.

BELL, S. M. (1970). The development of the concept of the object as related to infant-mother attachment. Child development, 41, 291-311.

Bowldy, J. (1969). Attachment and loss. Vol. 1: Attachment. Londres : Hogarth press.

BowlBY, J. (1980). Attachment and loss. Vol. 3: Loss, sadness and depression. Londres : Hogarth Press.

Brazelton, T.B.; Tronick, E.; Adamson, L.; Als, M. y WISE, S. (1975). Early mother-infant reciprocity. En Porter y O'Connor (Eds.) Mother-infant interaction. Amsterdam: Elsevier.

Brennan, W. M.; Ames, E. W., y Moore, R.W. (1966). Age differences in infants attention to patterns of different complexities. Science, 151, 354-355.

Bretherton, I.; Bates, E.; Benigni, L.; Camaion, L., y Volterra. V. (1979). Relationships betwen cognition, communication, and quality of attachment. En E. Bates (Ed.) The emergence of symbols. New york: Academic Press.

Bretherton, I. (1985). Attachment theory: Retrospect and prospect. En I. Bretherton y E. Waters (Eds.) Growing points of attachment theory and research. Monographs of the society for research in child development, 209, 50. Chicago: University of Chicago Press.

BRETherTON, I., y W ATERS, E. (1985). Growing points of attachment theory and research. Monographs of the society for research in child development. Chicago: University of Chicago Press.

BROSSARD, M.D. (1974). The infants conception of the object permanence and his reactions to strangers. En T. Gouin Decarie (Ed.) The infant's reactions to strangers. Nueva York: International Universities Press.

CARPENTER, G.C. (1975). Visual regard of moving and stationary faces in early infancy. Merrill-Palmer Quarterly 20, 181-194.

Cohon, J.F. y Tronick, E.Z. (1983). Three-month-old infants' reaction to simulated maternal depression. Child Development, 54, 185-193.

CORNELL, E.H. (1974). Infant's discrimination on faces following redundant presentations. Journal of Experimental Child psychology, 18, 98-106. 
DE CASPER, A.J. y FIfER, W.P. (1980). Of human bonding: newborns prefer their mother's voice. Science, 208, 1174-1176.

Diamond, A. (1985). Development of the ability to use recall to guide action, as indicated by infants_performance on AB. Child Development, 56, 868-883.

Dorse, W. (1978). Groups and individuals: Explanations in Social Psychology. Cambridge: Cambridge University Press

DoIse. W., y Mugny, G. (1979). Individual and collective conflicts of centrations in cognitive development. European Joumal of Social Psychology, 9, 105-109.

EISENBERG, R. B. (1976). Auditory Competence in early life: The roots of communicative behavior. Baltimore: University Park Press.

Fantz, R. L. (1961). The origin of form perception, Scientific American, 204, 66-72.

FANTZ, R. L. (1966). Patter discrimination and selective attention as determinants of perceptual development from birth. En A.H. Kidd y J.L. Rivoire (Eds.) Perceptual Development in Children. Nueva York: International Universities Press.

Fantz, R. L.; Fagan, J. F., y Miranda, S. B. (1975). Early visual selectivity. En L.B. Cohen y P.Salapatek (Eds.) Infant perception: From Sensation to Cognition. Nueva York: Academic Press.

FIELD, T. M. (1984). Early interactions between infants and their postpartum depressed mothers. Infant Behavior and Development, 7, 517-522.

Flavell, J. H. (1977). Cognitive development. New Jersey: Prentice Hall.

Gelman, R., y Spelke, E. (1981). The development of thoughts about animate and inanimate objects: Implications for research on social cognition. En J.H. Flavell y L. Ross (Eds.) Social cognitive development. Cambridge: Cambridge University Press.

Glachan, M., y Light (1982). Peer interaction and learning: Can two wrongs make a right ?. En G. Butterworth y P. Light (Eds.) Social Cognition. Studies of the Development of Understanding. Brighton: The Harvester Press Limited.

Gouin Decarie, T. (1962). Intelligence et afectivité chez le jeune enfant. Neuchatel: Delaclaux et Niestlé.

Gouin Decarie, T., y Ricard, M. (1983). La socialización del lactante. Mundo Científico, 22, 120-129. Hinde, R. A. (1966). Animal behavior: A synthesis of ethology and comparative psychology. Nueva York : McGraw-Hill.

Hutt, S. J.; Hutt, C.; Lenard. H. G.; Bernuth, H. V. y Muntjewerff, W.J. (1968). Audutory responsivity in the human neonate. Nature, 218, 888-890.

Isen, A. M.; Shalker. T.E.; Clarke, M., y Karp, L. (1978). Affect, accessibility of maternal in memory, and behavior: A cognitive loop? Joumal of Personality and Social Psychology, 36, 1-12.

KaGAN, J. (1974). Discrepancy, temperament, and infant distress. En Lewis, M. y Rosenblum, L.A. (Eds.) The origins of fear. Nueva York: Wiley.

KAGAN. J. (1984). The nature of the infant. Nueva York: Basic Books, Icn., Publisers.

Kagan. J.; Kearsley, R., y Zelazzo, P. (1978). Infancy: its place in human development. Cambridge, M.A. : Harvard University Press.

KAYE, K. (1972). Milk pressure as a determinant of the burst-pause pattern in neonatal sucking. Proceedings of th 80th Annual Convention of the American Psychological Association, 8, 83-84.

KAYE, K. (1977). Toward the origin of dialogue. En H. R. Schaffer (Ed.) Studies in motber-infant interaction. Londres: Academic Press.

KAYE, K. y WelLS, A. (1980). Mothers' jiggling and the burst-pause pattern in neonatal suckin. Infant Behavior and development, 3, 29-46.

Lecanuet.J. P.; Granier-Deferre.C., y Busnel. M. C. (1987). Familiarisation prénatale aux signaux de la parole. En M.Moscato, y G. Pieraut-Le Bonniec (Eds.) Connaître et le dire. Bruselas: Mardaga.

Maccoby, E. E., y Masters, J. C. (1970). Attachment and dependency. En P.H. Mussen (Ed.) Carmichael's manual of child psychology, Vol. 2. Nueva York : Wiley.

Macfarlane, J. A. (1975). Olfaction in the development of social preferences in the human neonate. En R. Porter y M. O'Connor (Eds.) The human neonate in parent-infant interaction. Amsterdam: Elsevier.

Main, M.; Kaplan, N., y Cassidy, J. (1985). Security in infancy, childhood and adulthood: a move to the level of representation. En I. Bretherton y E. Waters (Eds.) Growing points of attachment theory and researcb. Monographs of the society for research in child development, 209, 50. Chicago: University of Chirago Press.

Mandler.J. M. (1983). Representation. En J.H. Flavell y E.M. Markman (Eds.) P. H. Mussen (Series Ed.) Handbook of Child Psychology: Vol.3. Cognitive development. Nueva York: Wiley.

Mehler. J.; Bertoncini.J.; Barriere, M., y Jassik-Gerschienfeld, D. (1978). Infant recognition of mother voice. Perception, 7, 491-497.

Millar. W.S., y SCHAFFER, H.R. (1973). Visual-manipulative response strategies in infant operant conditioning with spatially displaced feedback. British Journal of Psychology, 64, 545-552

Montagner. H. (1988). L'attchment, les débuts de la tendresse. Paris: Editions Odile Jacob.

Nielson, I., y Dockrell, J. (1982). Cognitive tasks as interactional settings. En G. Butterworth y P. Light (Eds.) Social Cognition. Studies of the Development of Undentanding. Brighton: The Harvester Press Limited.

Olson, G. M. (1981). The recognition of specific persons. En M. E. Lamb y R. Sherrod (Eds.) Infant social cognition. Hillsdale, N.J.: L.Erlbaum. 
Palacios, J. (1984). Procesamiento de información en bebés. En J. Palacios, A. Marchesi y M. Carretero (Eds.) Psicología evolutiva. Vol. 2. Desarrollo cognitivo y social del niño. Madrid: Alianza Editorial.

Piaget. J. (1937). La construction du réel chez l'enfant. Neuchâtel-Paris: Delachaux- Niestlé.

Piaget. J. ( 1946). La formation du symbole chez l'enfant. Neuchâtel - Paris: Delachaux- Niestlé.

Rrviere, A. (1984). Acción e interacción en el origen del símbolo. En J. Palacios, A. Marchesi y M. Carretero (Eds.) Psicología evolutiva 2. Desarrollo cognitivo y social del niño. Madrid: Alianza.

Russell, M. J. (1976). Human olfactory communication. Nature, 260, 520.522.

Saint-Pierre. J. (1962). Etude des différences entre la recherche active de la personne humaine et celle de l'objet inanimé. Tesis doctoral no publicada, Universidad de Montreal. (Citado por Gouin Décarie, 1974)

SCHAFFER, H. R. (1966). The onset of fear of strangers and the incongruity hipothesis. Joumal of Child Psychology and Psychiatry, 7, 95-106.

Schaffer, H. R. (1971). The growth of Sociability. Hamodswort Middlesex: Penguin Books Ltd.

Schaffer, H. R. (1974). Cognitive components of the infant's response to strangeness. En Lewis, M. y Rosemblum, L.A. (Eds.): The origins of fear. New york: Academic Press.

Schank, R. C., y Abelson, R. (1977). Scripts, plans, goals and underrstanding. Hillsdale, N.J. : Lawrence Erlbaum Associates.

SpEARS, W. C. (1964). Assesment of visual preference and discrimination in the four-month-old infant. Joumal of Comparative Pbysiological Psychology, 57, 381-386

SROufe, L. A., y Waters (1977). Attachment as an organizatinal construct. Child Development, 48, 1184.1199

Sroufe, L. A. (1979). The ontogenesis of the emotions. En J. Osofsky (Ed.) Handbook of infant development. Nueva York: Wiley.

STERN, D. (1977). The first relationship: Infant and Motber. Londres: Open Books, ltd.

Trevarthen, C. (1977). Descriptive analysis of infantcommunicative behaviour. En H.R. Schaffer (Ed.) Studies in mother-infant interaction. Londres: Academic Press.

TREVARTHEN, C. (1979). Communication and cooperation in early infancy: A description of primary intersubjectivity. En M. Bullowa (Ed.) Before speech: The beginning of interpersonal communication. Cambridge: Cambridge University Press.

VIGotsky, L. S. (1962). Thought and Language. Cambridge, Massachusetts: MIT Press.

YarRow, L. (1967). The development of focused relationship during infancy. En J. Helmuth (Ed.) Exceptional Infant. Vol. 1. The Normal Infant. Seatle, Washington: Special Child Publications.

\section{Extended Summary}

Today the close relationship between cognitive and socio-emotional development is an accepted fact. In the subject under study - the cognitive construction of the infant's attachment figure- the need for an intergrated perspective is even more necessary.

The effort of a large number of researchers from the field of cognitive development to substantiate the social construction of social cognition in particular and of knowledge in general stands out. However, in relation to the origin and nature of the representation of the attachment figure, Piaget's structuralist view continues to charaterize developmental proposals.

The purpose of the present paper is first to analyze the explanatory capacity of Piaget's approach. The relationship between representation and symbolism proposed by Piaget implies that up to the end of the second year infants are able to recognize their attachment figure, but are unable to recall this figure in his/her abscence. The presence of anxiety reactions, when the infant is separated from the mother in the second half of the first year, and results from studies on the progression from recognition to recall suggest that there are differences between the representative and symbolic capacities both in their process and in their time of development. However, a strict application if the Piagetian process of construction of the physical reality in the social world appears limited and lacking. 
Secondly, the paper sought to defend and characterize: a) an interactive base in the construction of knowledge about the attachment figure; and b) the process by which a model of the mother figure is constructed on the basis of basic episodes of interaction (sensorymotor-emotional). In this model there are, on the one hand, perceptive elements and, on the other, associated experiences or expectations.

Finally, a type of representation known as an «Active Internal Model» (Bowlby) is described. This model includes both afective and cognitive components and it is built from the generalization of interactive episodes - of actions and consequences - relevant to the system of attachment. Once established, this representation is intergrated into the system of attachment exerting a determining influence on the focus of both attention and behaviour. This, therefore, allows us to addres individual differences on the security of attachment as differences on the representation of attachment. 